
\title{
R Research Square \\ Development and Validation of a Ferroptosis-related Prognostic Model in Pancreatic Cancer
}

\section{Chen-jie Qiu}

Nanjing Medical University

Xue-bing Wang

Nanjing Medical University

Zi-ruo Zheng

Nanjing Medical University

Chao-zhi Yang

Nanjing Medical University

Kai Lin

Nanjing Medical University

\section{Kai Zhang}

Nanjing Medical University

Min Tu

Nanjing Medical University

Kui-rong Jiang

Nanjing Medical University

Wentao Gao ( $\nabla$ gao11@hotmail.com )

Pancreas Institute, Nanjing Medical University, Nanjing, Jiangsu, China https://orcid.org/0000-00023332-8112

\section{Primary research}

Keywords: Ferroptosis, Pancreatic cancer, Prognostic model, Bioinformatics, Immune cell infiltration

Posted Date: January 7th, 2021

DOl: https://doi.org/10.21203/rs.3.rs-139597/v1

License: (c) (i) This work is licensed under a Creative Commons Attribution 4.0 International License. Read Full License 


\section{Abstract}

Background: With the development of genomics, ferroptosis has been determined to be highly important in cancer. The purpose of this study was to identify ferroptosis-related genes (FRGs) associated with the prognosis of pancreatic cancer and to construct a prognostic model based on FRGs.

Methods: Based on pancreatic cancer data obtained from The Cancer Genome Atlas (TCGA) database, we employed univariate Cox analysis, least absolute shrinkage and selection operator (LASSO) analysis and multivariate Cox analysis to establish the prognostic model from $232 \mathrm{FRGs}$. A nomogram was constructed by combining the prognostic model and clinicopathological features. Gene Expression Omnibus (GEO) datasets and tissue samples obtained from our center were utilized to validate the prognostic model. Relationship between risk score and immune cell infiltration was explored by CIBERSORT and TIMER.

Results: The prognostic model was established based on four FRGs (ENPP2, ATG4D, SLC2A1 and MAP3K5) and can be an independent risk factor in pancreatic cancer (HR 1.648, 95\% Cl 1.335-2.035, $\mathrm{p}<$ 0.001). Based on the median risk score, patients were divided into a high-risk group and a low-risk group. The KM curve indicated that the overall survival (OS) of the low-risk group was significantly better than that of the high-risk group. The nomogram showed that the prognostic model was the most important element. Gene set enrichment analysis (GSEA) identified three key pathways, namely, TGF $\beta$ signaling, HIF signaling pathway and adherens junction. GSE57495, GSE62452 and 88 pancreatic cancer tissues from our center were utilized to validate the prognostic model. The prognostic model can also affect the immune cell infiltration, such as macrophages M0, M1, CD4+T cell and CD8+T cell.

Conclusion: A ferroptosis-related prognostic model can be employed to predict the prognosis of pancreatic cancer. Ferroptosis can be an important marker and immunotherapy can be a potential therapeutic target for pancreatic cancer.

\section{Background}

Pancreatic cancer is a common malignant tumor of the pancreas, with a poor prognosis. The 5-year survival rate of pancreatic cancer is approximately $9 \%$ in the United States, and the median survival time in China is less than 7.8 months[1-3]. The morbidity and mortality of pancreatic cancer have increased in recent years[1]. Studies have shown that by 2030, pancreatic cancer will be the second leading cause of cancer-related deaths $[4,5]$. One of the primary causes of the poor prognosis of pancreatic cancer is delayed diagnosis. Due to the deep anatomical location of the pancreas and hidden clinical manifestations of these cancers in the early stages, most pancreatic cancers are detected in the advanced stages when the opportunity for surgery has passed[3]. In the past decade, with the development of genomics, the diagnosis and treatment of pancreatic cancer patients have been considerably improved. 
Ferroptosis is an iron-dependent nonapoptotic form of cell death that is characterized by excessive accumulation of lipid peroxides and reactive oxygen species (ROS)[6]. Ferroptosis is identified to be closely related to a series of different tumor types[6], such as hepatocellular carcinoma[7, 8], prostate cancer[9], breast cancer[10], and pancreatic cancer[11-13]. This type of cell death can inhibit the proliferation of tumor cells and kill tumor cells as a new tumor inhibition pathway[14, 15]. Recent studies[13] have determined that artesunate plays an anticancer role in pancreatic cancer by affecting ferroptosis. Another study[16] showed that piperlongumine was highly effective for pancreatic cancer, as it can significantly increase intracellular ROS levels, induce ferroptosis and be inhibited by ferroptosis inhibitors and the iron chelator deferoxamine. Numerous genes have also been identified as drivers, suppressors or markers of ferroptosis. Ferroptosis-related genes (FRGs), such as HSPA5, can act as antitumor agents for gemcitabine by inducing ferroptosis[17]. Also, knocking out or knocking down ATG5 and ATG7 was observed to inhibit ferroptosis in pancreatic cancer cells[18].

With the development of sequencing technology, it has become possible to study FRGs and clinical features to build prognostic models. Characterizing the molecular landscape of pancreatic cancer may help to stratify patients by risk and adopt different treatment plans in a personalized way to maximize the benefits for patients[19]. To determine the significance of FRGs for pancreatic cancer patients, we screened out the FRGs significantly related to the overall survival (OS) of pancreatic cancer patients to construct a prognostic model and verify its reliability with external datasets. In addition, we analyzed the correlation between prognosis-related FRGs and clinicopathological parameters, and constructed a nomogram based on clinicopathological parameters and a prognostic model. We also evaluated the correlation between risk score and immune cell infiltration and studied immune checkpoints to seek immunotherapy for pancreatic cancer.

\section{Methods}

\section{Data source}

RNA sequencing data and relevant clinical information for pancreatic cancer, such as age, gender, grade, stage, TNM stage and survival, were downloaded from The Cancer Genome Atlas (TCGA, https://portal.gdc.cancer.gov/). A list of up-to-date FRGs was downloaded from the FerrDb database (http://www.zhounan.org/ferrdb). The GSE62452 and GSE57495 datasets were obtained from the Gene Expression Omnibus (GEO, https://www.ncbi.nIm.nih.gov/geo/) for validation.

\section{Identification of differentially expressed FRGs (DE-FRGs)}

Perl software was used to obtain the expression levels of FRGs in TCGA samples. Genes with p-value < 0.05 and $\mid \log$ FoldChange| $>1$ were considered to be DE-FRGs. The DE-FRGs were visualized by the $R$ packages "volcano" and "heatmap". The functional enrichment analyses of DE-FRGs primarily involving Gene Ontology (GO) terms and Kyoto Encyclopedia of Genes and Genomes (KEGG) pathway analysis were performed with the R package "clusterprofiler". GO analysis included the functions of DE-FRGs in biological processes (BP), cellular components (CC), and molecular functions (MF), and KEGG analysis 
demonstrated the pathway enrichment of DE-FRGs. The STRING database (https://string-db.org/) was utilized to construct a protein-protein interaction (PPI) network. Next, we input the interacting data derived from STRING to Cytoscape for visualization. The correlation between the DE-FRGs was calculated by Pearson's test and visualized by the R package "corrplot".

\section{Identification of prognostic-related genes and construction of a prognostic model}

Univariate Cox analysis was employed to identify FRGs related to the OS of pancreatic cancer patients. The OS-related FRGs were included in least absolute shrinkage and selection operator (LASSO) analysis to be further screened. Multivariate Cox analysis was further used to identify independent risk FRGs associated with prognosis. Finally, a prognostic model was constructed via the linear combination of the expression levels of FRGs, with the multivariate Cox regression coefficient $(\beta)$ serving as the weight. The prognostic model was as follows: risk score $=$ expression of gene $1 \times \beta 1+$ expression of gene $2 \times \beta 2+\ldots+$ expression of gene $n \times \beta n$. Patients were divided into a high-risk group and a low-risk group according to the median risk score. The Kaplan-Meier (KM) method was employed to describe the prognostic difference between the two groups. To judge the feasibility of the prognostic model constructed above, we incorporated risk score, age, gender, stage, grade, $\mathrm{T}$ stage and $\mathrm{N}$ stage into univariate and multivariate Cox regression analyses to determine independent risk factors. We also incorporated these factors into receiver operating characteristic (ROC) analysis to assess the accuracy and sensitivity of the model. The GSE62452 and GSE57495 datasets were chosen to validate the prognostic model finally. The X-tile software was used to determine the best cutoff value for OS.

\section{Construction of nomogram}

The R packages "rms" and "survival" were used to construct the nomogram based on the Cox regression model. Calibration curves were described to assess the consistency between actual and predicted 1-year, 2-year and 3-year survival to further judge the prognostic model.

\section{Experimental verification}

A total of 88 pancreatic cancer tissues were obtained from the pancreatic center of the First Affiliated Hospital of Nanjing Medical University. The tissues were frozen in liquid nitrogen after surgery was performed. The study was approved by the Ethics Committee of the First Affiliated Hospital of Nanjing Medical University (No. 2018-SR-344.A2). Total RNA was isolated with TRIzol (Takara, Japan) according to the manufacturer's instructions and subsequently reverse-transcribed into cDNA through PrimeScript RT MasterMix (Takara, Japan). Real Time Quantitative Polymerase Chain Reaction (RT-qPCR) was performed with SYBR Green (Vazyme, China). The levels of ENPP2, ATG4D, SLC2A1 and MAP3K5 were normalized to $18 \mathrm{~S}$ rRNA and calculated using the $2^{-\triangle \Delta C t}$ method. The primers are shown in Table S1.

\section{Gene set enrichment analysis (GSEA) in the high-risk group}


Based on the criterion of $p$-value $<0.05$ and |log FoldChange| $>1$, difference genes between the high-risk and the low-risk group were found and were performed enrichment analysis using Metascape (http://metascape.org/gp/index.html) to predict the potential mechanisms in the high-risk group. In addition, GSEA (http://www.broadinstitute.org/gsea) was applied to the Molecular Signatures Database v7.2, including $\mathrm{H}$ : hallmark gene sets and C2: curated gene sets (BIOCARTA and KEGG), to identify enriched pathways related to the high-risk group. The enriched gene sets were obtained based on $\mathrm{p}$ values $<0.05$ and false discovery rate $($ FDR ) values $<0.25$.

\section{Relationship between immune cell infiltration and risk score}

R package "CIBERSORT" and "ESTIMATE" was utilized to calculate the immune score, stromal score and estimate score of the tumor samples in pancreatic cancer of TCGA based on 22 types of immune cells. The immune cell infiltration and somatic number alteration (SCNA) were also assessed by Tumor Immune Estimation Resource (TIMER, https://cistrome.shinyapps.io/timer/) based on six immune cells, including B cell, CD8+T cell, CD4+T cell, macrophage, neutrophil and dendritic cell. Finally, we evaluated the relationship between risk score and classical immune checkpoints, such as PDCD1, CTLA4 and CD274 with Pearson's test.

\section{Results}

\section{Identification and enrichment analysis of DE-FRGs}

The gene expression profiles and clinical information of 182 cases, including 178 tumor cases and 4 normal cases, were downloaded from TCGA database. Patients with a survival time $<=30$ days were excluded from subsequent analysis. The clinical information of pancreatic cancer patients is presented in Table S2. A total of 232 FRGs were selected from the FerrDb database. After extracting the gene expression of FRGs from TCGA samples, we performed the differential analysis. According to the standards mentioned above, 18 DE-FRGs were acquired by the Wilcoxon test, among which 10 were upregulated, while 8 were downregulated (Fig. 1A-1B). Next, we performed GO and KEGG pathway analyses to further study the biological functions of ferroptosis in the development of pancreatic cancer. GO analysis indicated that these DE-FRGs were enriched in biological processes related to ferroptosis, such as cellular response to oxidative stress, chemical stress, toxic stress, response to oxidative stress and liver development. The cellular components and molecular functions of these DE-FRGs are shown in Fig. 1C. KEGG pathway analysis suggested that most of the DE-FRG pathways were significantly linked to ferroptosis, leishmaniasis, HIF-1 signaling pathway, fluid shear stress and atherosclerosis and phagosomes (Fig. 1D). Using STRING and Cytoscape software, we constructed a PPI network for these DE-FRGs (Fig. 1E). The correlation of these DE-FRGs is presented in Fig. 1F.

\section{Construction of FRGs-based prognostic model}

All FRGs were included in univariate Cox regression analysis for construction of the prognostic model. The results of univariate Cox analysis showed that 13 FRGs were associated with the prognosis of 
pancreatic cancer patients (Fig. 2A). The relative expression levels of the 13 genes in tumor and normal samples are shown in Fig. 2B. The 13 genes were subsequently input into LASSO regression to narrow the genes and effectively avoid overfitting (Fig. 2C-2D). Finally, the selected genes were analyzed by multivariate Cox regression analysis, and 4 genes were obtained.

Risk score $=\left(-0.30685^{\star}\right.$ ENPP2 $)+\left(-0.85263^{\star}\right.$ ATG4D $)+(0.119446 \star S L C 2 A 1)+(0.636851 *$ MAP3K5 $)$.

The median risk score was applied to divide the pancreatic cancer patients into a high-risk group or a lowrisk group. Fig. 3A shows the risk score of each pancreatic cancer patient and Fig. 3B shows the relationship between the risk score and survival time. Fig. $3 \mathrm{C}$ shows the gene expression profiles of the four genes in the high-risk group and low-risk group by heatmap.

\section{Prognostic model as an independent risk factor}

We evaluated the clinical and pathological parameters and risk score for prognostic value by univariate and multivariate analyses. Fig. 4A demonstrates that age, $\mathrm{N}$ stage and risk score were associated with the OS, and Fig. 4B shows that the risk score can become an independent risk factor (HR 1.648,95\% Cl 1.335-2.035, $\mathrm{p}<0.001$ ). In addition, the KM curve showed that the high-risk group had a poorer OS rate than did the low-risk group (Fig. 4C). ROC analysis was performed to explore the sensitivity and specificity of the model and the area under the curve (AUC) was 0.673 , which was higher than that of other clinical and pathological parameters (Fig. 4D). In addition, the patients were stratified based on clinicopathologic parameters for further survival analysis. In almost all stratified analyses, this risk model can achieve good performance, except for stage III\&IV and T1\&2, which may be attributable to the low number of cases (Fig.S1).

\section{Correlation between prognostic model and clinicopathological characteristics}

Based on the association of these FRGs with OS in pancreatic cancer, we further examined the correlation between the gene expression levels of FRGs and clinicopathological characteristics, such as age $(>=65 /<$ 65), gender (male/female), grade (G3\&4/G1\&2), stage (stage III\&IV/stage I\&II), T stage (T3\&4/T1\&2) and $\mathrm{N}$ stage (N1/N0). Only ATG4D was significantly associated with stage and N stage (Fig. S2A-S2B), and the risk score was closely related to T stage (Fig. S2C).

\section{Construction of the nomogram for prediction of prognostic risk}

To better determine the survival risk of pancreatic cancer patients, we integrated the prognostic model and clinicopathological features to construct a nomogram. A nomogram was constructed to predict the 1-year, 3-year and 5-year survival possibilities (Fig. 5A). The calibration curve showed that when compared with the actual 1-year, 2-year and 3-year survival rates, the survival rates predicted by the nomogram were able to achieve good agreement (Fig. 5B-5D).

\section{Validation of the prognostic model}


To validate the prognostic model, a total of 88 pancreatic cancer tissue samples from our center were used for further experimental verification. The KM curve showed that the OS of the low-risk group was notably better than that of the high-risk group (Fig. 6A). Fig. 6B shows that the AUC of the prognostic model was 0.647 . Fig. $6 \mathrm{C}$ shows that the higher the risk score was, the lower the survival time was. The heatmap of the 4 genes is also shown in Fig. $6 \mathrm{C}$. The nomogram was validated, and the 1-year and 1.5year calibration curves are shown in Fig. 6D.

In addition, GSE57495 and GSE62452 datasets were downloaded for further assessment. Fig. S3 validates the prognostic model in GSE57495 and GSE62452.

\section{Enrichment analyses in the high-risk group}

To further study the enriched pathways in the high- and low-risk groups, the differential analysis was performed and the differential expressed genes were input into the online tool Metascape (Fig.7A). The enrichment analysis showed that the genes enriched in several biological functions, such as chemical synaptic transmission, regulation of ion transport, regulation of system process, neuronal system and inorganic cation transmembrane transport (Fig. 7B-7D). In addition, GSEA showed that the pathways enriched in the high-risk group were TGF $\beta$ signaling, HIF signaling pathway and adherens junction (Fig. 7E).

\section{Immune landscape between the high- and low-risk groups}

We assessed the infiltration degree of 22 types of immune cells in the high-risk and low-risk groups, and calculated the immune score and immune microenvironment score. Fig. 8A showed the immune cells, immune score, stromal score and estimate score in pancreatic cancer patients and risk score was significantly associated with immune score. Fig. 8B revealed that some immune cells differed significantly between high- and low-risk groups, indicating that immune cells played an important role. Macrophage $\mathrm{M} 0$ and $\mathrm{M} 1$ showed higher infiltration in high-risk group. To further examinate the relationship between the risk score and immune cell infiltration, the four risk genes were firstly studied by TIMER. ENPP2 expression was associated with B cell, CD8+T cell, CD4+T cell, macrophage, neutrophil and dendritic cell, and the other three genes were also related to some of the immune cells (Fig. $8 \mathrm{C}$ ). Figure 8D-81 revealed that the risk score was negatively correlated with CD4+T cell $(p=0.009, R=-0.199)$ and positively correlated with CD8+T cell $(p=0.033, R=0.163)$. In addition, the relationship between SCNA of the four genes and immune cell infiltration was explored by TIMER. Fig. S4 revealed that SCNA may result in changes of immune infiltration levels of $B$ cell and $C D 4+T$ cell.

\section{Relationship between immune checkpoints and risk score}

Immunotherapy played an important role in pancreatic cancer. In this study, we evaluated the relationship between risk score and classical immune checkpoints, such as PDCD1, CTLA4 and CD274. As shown in Fig. 9, immune checkpoints were correlated to risk score, as were the four genes included in the 
prognostic model. Fig. 9C revealed that CD274 may be a key immune checkpoint in pancreatic cancer treatment.

\section{Discussion}

In this study, we first obtained FRGs through the ferroptosis database FerrDb. We analyzed their gene expression levels from the TCGA database. Univariate Cox regression analysis showed that 13 FRGs were associated with the prognosis of pancreatic cancer. The signature of four genes was established by LASSO regression and multivariate Cox analysis. The risk score of each patient was calculated by multiplying the risk coefficient by the expression level of the four genes. Through survival analysis, we observed that the prognostic model can achieve good performance for all cohorts or stratified analysis, except stages III\&IV and T1\&2. The low performance in stages III\&IV and T1\&2 may be due to the small number of cases. In addition, univariate and multivariate Cox analyses showed that the risk score could be an independent risk factor for pancreatic cancer. We further studied the correlation between the prognostic model and clinicopathological parameters, and the risk score was demonstrated to be highly associated with $\mathrm{T}$ stage. Finally, two independent GEO datasets and 88 pancreatic cancer samples in our center were selected to validate the prognostic performance.

At present, few studies have reported prognostic models of FRGs. These models have only has been reported in liver cancer[20], clear cell renal cell carcinoma[21] and glioma[22]. However, the sources of the FRGs that these studies employed obtained were only from a comprehensive literature survey, which may lead to the loss of FRGs. For example, Liang el al.[20] identified 60 FRGs from the previous literature and conducted a 10-FRG signature to predict the prognosis of liver cancer.

The four FRGs in the prognostic model were identified in FerrDb and they could be classified into three categories: markers (SLC2A1 and MAP3K5), suppressors (ENPP2) and drivers (ATG4D). SLC2A1 encodes the GLUT-1 protein, a glucose transporter member[23]. Previous studies have indicated that GLUT-1 is a prognostic protein for pancreatic cancer patients[24-26]. GLUT-1 is highly expressed in pancreatic cancer and promotes the proliferation of pancreatic cancer cells[24]. LSH interacted with WDR76 to inhibit ferroptosis by activating the lipid metabolism-associated gene GLUT-1[27]. ENPP2, termed ATX, can produce the signaling molecule lysophosphatidic acid (LPA) as a secreted enzyme, and the lipid metabolic reaction may be associated with the development of colorectal cancer[28, 29]. ENPP2/LPA signaling can significantly inhibit ROS generation and ferroptosis in cardiomyocytes by regulating GPX4, ACSL4 and NRF2[30]. The expression of ATX was elevated in pancreatic cancer by microarray analysis, and serum ATX activity might be a useful marker[31, 32]. Many autophagy-related genes, including ATG4D, were identified as positive regulators of ferroptosis by RNAi screening and genetic analysis[33], and this study showed that autophagy played a crucial role in ferroptosis. ATG4D was regulated by SNHG14 and miR-101 in gemcitabine resistance and cell viability in pancreatic cancer. MAP3K5, also known as ASK1, is a serine/threonine kinase that plays an important role in the MAP kinase signal transduction pathway[34]. ASK1 is activated by cytotoxic stresses, such as lipopolysaccharide (LPS) and ROS[34]. ASK1 acts as an oncogene in the development of pancreatic cancer by upregulating cyclin E. 
ASK1 is also critical for LPS-induced p38 activation and cytokine production[35]. Previous studies have shown that cold stress can induce ferroptosis and the ASK1-p38 MAPK pathway. The ASK1-p38 pathway is a regulator of ferroptosis downstream of lipid peroxide[36].

Based on DE-FRGs between the normal and tumor groups, we performed KEGG pathway analysis, and HIF-1 signaling pathway was enriched. At the same time, the HIF signaling pathway was enriched in the high-risk group by GSEA. HIF-1 signaling pathway can play an important role in the carcinogenesis and progression of pancreatic cancer and drive ferroptosis sensitivity in cancers[37, 38]. Jiang et al.[27] found that LSH interacted with WDR76 to inhibit ferroptosis and that the expression of LSH was activated by EGLN1 and c-Myc by inhibiting HIF-1a. Yang et al.[39] showed that the degradation of ARNTL can promote ferroptosis by promoting the expression of EGLN2, thereby destabilizing HIF-1a.

We constructed a nomogram to predict the prognosis of pancreatic cancer patients. The nomogram, which included age, gender, grade, stage, $\mathrm{T}$ stage, $\mathrm{N}$ stage and risk score, showed that the risk score was the most important variable. Moreover, the calibration plot showed that actual survival and predicted survival can achieve good agreement, indicating that the prognostic model can accurately assess prognosis.

We found that the prognostic model can not only be associated with the OS, but also affect the immune infiltration level of pancreatic cancer patients. Based on CIBERSORT and ESTIMATE algorithm, the infiltration level of macrophages M0 and M1 was increased in the high-risk group. Previous studies showed that PD-L1 expression was positive in about $30 \%$ of pancreatic cancer patients[40]. Sporadic clinical evidence suggested that PD-1/PD-L1 monotherapy was almost ineffective in pancreatic cancer patients[41]. Recent studies have found that PD-1/PD-L1 inhibitors combined with other immunotherapies, such as PD-1 combined with Galectin-9 antibody[42], can significantly inhibit the growth of pancreatic cancer cells. Hence, targeted therapy and immune checkpoints therapy for pancreatic cancer will be a hot topic in the future.

However, our study also has several limitations. First, this study is a retrospective study, and the model should be validated with prospective data. Second, we did not include $M$ stage in the nomogram due to missing $M$ stage data.

\section{Abbreviations}

FRG: ferroptosis-related genes; DE-FRG: differentially expressed ferroptosis-related genes; TCGA: The Cancer Genome Atlas; LASSO: least absolute shrinkage and selection operator; GEO: Gene Expression Omnibus; OS: overall survival; GSEA: Gene set enrichment analysis; ROS: reactive oxygen species; GO: Gene Ontology; KEGG: Kyoto Encyclopedia of Genes and Genomes; BP: biological processes; CC: cellular components; MF: molecular functions; PPI: protein-protein interaction; KM: Kaplan-Meier; ROC: receiver operating characteristic; AUC: the area under the curve; 
RT-qPCR: Real Time Quantitative Polymerase Chain Reaction; FDR: false discovery rate; SCNA: somatic number alteration; TIMER: Tumor Immune Estimation Resource

\section{Conclusion}

We conducted and validated a prognostic model of four ferroptosis-related genes. A nomogram that included the risk score and clinicopathological parameters was also established to predict the OS of pancreatic cancer patients. Immune checkpoints inhibitor can be a potential therapy for pancreatic cancer.

\section{Declarations}

\section{Acknowledgements}

Not applicable.

\section{Authors' contributions}

Chen-jie Qiu and Xue-bing Wang carried out the experimental design, data analysis. Zi-ruo Zheng wrote the manuscript. All authors read and approved the final manuscript.

\section{Funding}

This work was supported by Wu jieping medical foundation (Grant/Award Number: 320.2710.1802).

\section{Availability of data and materials}

All data generated or analyzed during this study are included in this published article and its supplementary information files.

\section{Ethics approval and consent to participate}

The study was approved by the Ethics Committee of the First Affiliated Hospital of Nanjing Medical University.

\section{Consent for publication}

No individual person's data in any form were involved in this study.

\section{Competing Interests}

The authors declare that there is no conflict of interests.

\section{References}


1. Siegel RL, Miller KD, Jemal A: Cancer statistics, 2020. CA Cancer J Clin 2020, 70(1):7-30.

2. Bray F, Ferlay J, Soerjomataram I, Siegel RL, Torre LA, Jemal A: Global cancer statistics 2018: GLOBOCAN estimates of incidence and mortality worldwide for 36 cancers in 185 countries. CA Cancer J Clin 2018, 68(6):394-424.

3. Mizrahi JD, Surana R, Valle JW, Shroff RT: Pancreatic cancer. Lancet 2020, 395(10242):2008-2020.

4. Rahib L, Smith BD, Aizenberg R, Rosenzweig AB, Fleshman JM, Matrisian LM: Projecting cancer incidence and deaths to 2030: the unexpected burden of thyroid, liver, and pancreas cancers in the United States. Cancer Res 2014, 74(11):2913-2921.

5. Neoptolemos JP, Kleeff J, Michl P, Costello E, Greenhalf W, Palmer DH: Therapeutic developments in pancreatic cancer: current and future perspectives. Nat Rev Gastroenterol Hepatol 2018, 15(6):333348.

6. Dixon SJ, Lemberg KM, Lamprecht MR, Skouta R, Zaitsev EM, Gleason CE, Patel DN, Bauer AJ, Cantley AM, Yang WS et al: Ferroptosis: an iron-dependent form of nonapoptotic cell death. Cell 2012, 149(5):1060-1072.

7. Louandre C, Marcq I, Bouhlal H, Lachaier E, Godin C, Saidak Z, Francois C, Chatelain D, Debuysscher $\mathrm{V}$, Barbare JC et al: The retinoblastoma (Rb) protein regulates ferroptosis induced by sorafenib in human hepatocellular carcinoma cells. Cancer Lett 2015, 356(2 Pt B):971-977.

8. Zhang X, Du L, Qiao Y, Zhang X, Zheng W, Wu Q, Chen Y, Zhu G, Liu Y, Bian Z et al: Ferroptosis is governed by differential regulation of transcription in liver cancer. Redox Biol 2019, 24:101211.

9. Dixon SJ, Patel DN, Welsch M, Skouta R, Lee ED, Hayano M, Thomas AG, Gleason CE, Tatonetti NP, Slusher BS et al: Pharmacological inhibition of cystine-glutamate exchange induces endoplasmic reticulum stress and ferroptosis. Elife 2014, 3:e02523.

10. Mao C, Wang X, Liu Y, Wang M, Yan B, Jiang Y, Shi Y, Shen Y, Liu X, Lai W et al: A G3BP1-Interacting IncRNA Promotes Ferroptosis and Apoptosis in Cancer via Nuclear Sequestration of p53. Cancer Res 2018, 78(13):3484-3496.

11. Lachaier E, Louandre C, Godin C, Saidak Z, Baert M, Diouf M, Chauffert B, Galmiche A: Sorafenib induces ferroptosis in human cancer cell lines originating from different solid tumors. Anticancer Res 2014, 34(11):6417-6422.

12. Kasukabe T, Honma Y, Okabe-Kado J, Higuchi Y, Kato N, Kumakura S: Combined treatment with cotylenin A and phenethyl isothiocyanate induces strong antitumor activity mainly through the induction of ferroptotic cell death in human pancreatic cancer cells. Oncol Rep 2016, 36(2):968-976.

13. Eling N, Reuter L, Hazin J, Hamacher-Brady A, Brady NR: Identification of artesunate as a specific activator of ferroptosis in pancreatic cancer cells. Oncoscience 2015, 2(5):517-532.

14. Torii S, Shintoku R, Kubota C, Yaegashi M, Torii R, Sasaki M, Suzuki T, Mori M, Yoshimoto Y, Takeuchi $\mathrm{T}$ et al: An essential role for functional lysosomes in ferroptosis of cancer cells. Biochem J 2016, 473(6):769-777.

15. Yu H, Guo P, Xie X, Wang Y, Chen G: Ferroptosis, a new form of cell death, and its relationships with tumourous diseases. J Cell Mol Med 2017, 21(4):648-657. 
16. Yamaguchi Y, Kasukabe T, Kumakura S: Piperlongumine rapidly induces the death of human pancreatic cancer cells mainly through the induction of ferroptosis. Int J Oncol 2018, 52(3):10111022.

17. Zhu S, Zhang Q, Sun X, Zeh HJ, 3rd, Lotze MT, Kang R, Tang D: HSPA5 Regulates Ferroptotic Cell Death in Cancer Cells. Cancer Res 2017, 77(8):2064-2077.

18. Hou W, Xie Y, Song X, Sun X, Lotze MT, Zeh HJ, 3rd, Kang R, Tang D: Autophagy promotes ferroptosis by degradation of ferritin. Autophagy 2016, 12(8):1425-1428.

19. Yan J, Wu L, Jia C, Yu S, Lu Z, Sun Y, Chen J: Development of a four-gene prognostic model for pancreatic cancer based on transcriptome dysregulation. Aging (Albany NY) 2020, 12(4):3747-3770.

20. Liang JY, Wang DS, Lin HC, Chen XX, Yang H, Zheng Y, Li YH: A Novel Ferroptosis-related Gene Signature for Overall Survival Prediction in Patients with Hepatocellular Carcinoma. Int J Bio/ Sci 2020, 16(13):2430-2441.

21. Wu G, Wang Q, Xu Y, Li Q, Cheng L: A new survival model based on ferroptosis-related genes for prognostic prediction in clear cell renal cell carcinoma. Aging (Albany NY) 2020, 12(14):1493314948.

22. Liu HJ, Hu HM, Li GZ, Zhang Y, Wu F, Liu X, Wang KY, Zhang CB, Jiang T: Ferroptosis-Related Gene Signature Predicts Glioma Cell Death and Glioma Patient Progression. Front Cell Dev Bio/ 2020, 8:538.

23. Augustin R: The protein family of glucose transport facilitators: It's not only about glucose after all. IUBMB Life 2010, 62(5):315-333.

24. Cheng Y, Wang K, Geng L, Sun J, Xu W, Liu D, Gong S, Zhu Y: Identification of candidate diagnostic and prognostic biomarkers for pancreatic carcinoma. EBioMedicine 2019, 40:382-393.

25. Nagarajan A, Dogra SK, Sun L, Gandotra N, Ho T, Cai G, Cline G, Kumar P, Cowles RA, Wajapeyee N: Paraoxonase 2 Facilitates Pancreatic Cancer Growth and Metastasis by Stimulating GLUT1Mediated Glucose Transport. Mol Cel/ 2017, 67(4):685-701 e686.

26. Yu M, Zhou Q, Zhou Y, Fu Z, Tan L, Ye X, Zeng B, Gao W, Zhou J, Liu Y et al: Metabolic phenotypes in pancreatic cancer. PLoS One 2015, 10(2):e0115153.

27. Jiang Y, Mao C, Yang R, Yan B, Shi Y, Liu X, Lai W, Liu Y, Wang X, Xiao D et al: EGLN1/c-Myc Induced Lymphoid-Specific Helicase Inhibits Ferroptosis through Lipid Metabolic Gene Expression Changes. Theranostics 2017, 7(13):3293-3305.

28. Perrakis A, Moolenaar WH: Autotaxin: structure-function and signaling. J Lipid Res 2014, 55(6):10101018.

29. Zhao Z, Xiao Y, Elson P, Tan H, Plummer SJ, Berk M, Aung PP, Lavery IC, Achkar JP, Li L et al: Plasma lysophosphatidylcholine levels: potential biomarkers for colorectal cancer. J Clin Oncol 2007, 25(19):2696-2701.

30. Bai YT, Chang R, Wang H, Xiao FJ, Ge RL, Wang LS: ENPP2 protects cardiomyocytes from erastininduced ferroptosis. Biochem Biophys Res Commun 2018, 499(1):44-51. 
31. Nakai Y, Ikeda H, Nakamura K, Kume Y, Fujishiro M, Sasahira N, Hirano K, Isayama H, Tada M, Kawabe $\mathrm{T}$ et al: Specific increase in serum autotaxin activity in patients with pancreatic cancer. Clin Biochem 2011, 44(8-9):576-581.

32. Quan M, Cui JJ, Feng X, Huang Q: The critical role and potential target of the autotaxin/lysophosphatidate axis in pancreatic cancer. Tumour Bio/2017, 39(3):1010428317694544.

33. Gao M, Monian P, Pan Q, Zhang W, Xiang J, Jiang X: Ferroptosis is an autophagic cell death process. Cell Res 2016, 26(9):1021-1032.

34. Luo Y, Gao S, Hao Z, Yang Y, Xie S, Li D, Liu M, Zhou J: Apoptosis signal-regulating kinase 1 exhibits oncogenic activity in pancreatic cancer. Oncotarget 2016, 7(46):75155-75164.

35. Matsuzawa A, Saegusa K, Noguchi T, Sadamitsu C, Nishitoh H, Nagai S, Koyasu S, Matsumoto K, Takeda K, Ichijo H: ROS-dependent activation of the TRAF6-ASK1-p38 pathway is selectively required for TLR4-mediated innate immunity. Nat Immuno/ 2005, 6(6):587-592.

36. Hattori K, Ishikawa H, Sakauchi C, Takayanagi S, Naguro I, Ichijo H: Cold stress-induced ferroptosis involves the ASK1-p38 pathway. EMBO Rep 2017, 18(11):2067-2078.

37. Jin X, Dai L, Ma Y, Wang J, Liu Z: Implications of HIF-1alpha in the tumorigenesis and progression of pancreatic cancer. Cancer Cell Int 2020, 20:273.

38. Zou Y, Palte MJ, Deik AA, Li H, Eaton JK, Wang W, Tseng YY, Deasy R, Kost-Alimova M, Dancik V et al: A GPX4-dependent cancer cell state underlies the clear-cell morphology and confers sensitivity to ferroptosis. Nat Commun 2019, 10(1):1617.

39. Yang M, Chen P, Liu J, Zhu S, Kroemer G, Klionsky DJ, Lotze MT, Zeh HJ, Kang R, Tang D: Clockophagy is a novel selective autophagy process favoring ferroptosis. Sci Adv 2019, 5(7):eaaw2238.

40. Hu G, He N, Cai C, Cai F, Fan P, Zheng Z, Jin X: HDAC3 modulates cancer immunity via increasing PDL1 expression in pancreatic cancer. Pancreatology 2019, 19(2):383-389.

41. Feng $M$, Xiong G, Cao Z, Yang G, Zheng S, Song X, You L, Zheng L, Zhang T, Zhao Y: PD-1/PD-L1 and immunotherapy for pancreatic cancer. Cancer Lett 2017, 407:57-65.

42. Wang S, Yao F, Lu X, Li Q, Su Z, Lee JH, Wang C, Du L: Temozolomide promotes immune escape of GBM cells via upregulating PD-L1. Am J Cancer Res 2019, 9(6):1161-1171.

\section{Figures}



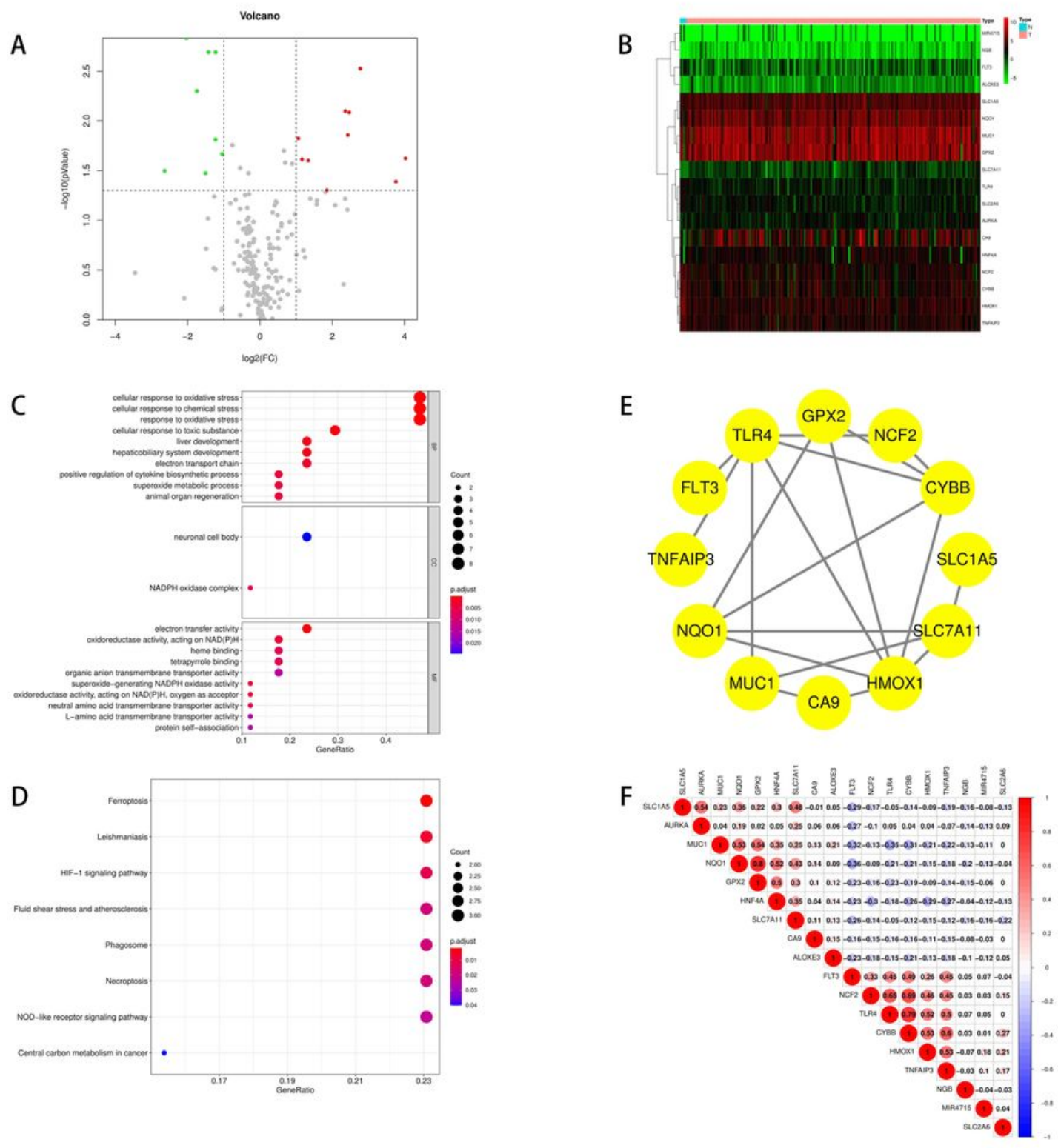

\section{Figure 1}

Identification and enrichment analysis of DE-FRGs. A Volcano plot of 232 FRGs in TCGA database. The criteria were $p<0.05$ and $|\log F C|>1$. B Heatmap of 18 DE-FRGs in TCGA database. C GO analysis of DEFRGs, including BP (biological process), CC (cellular component) and MF (molecular function). D KEGG pathway analysis. E Protein-protein interaction network. F Correlation analysis of DE-FRGs. 
A
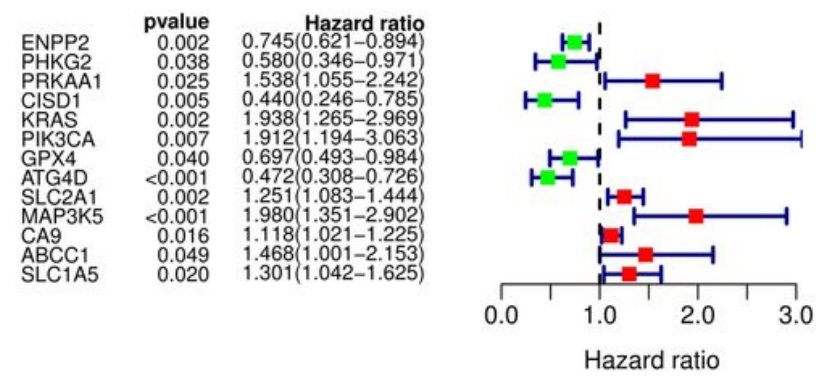

C

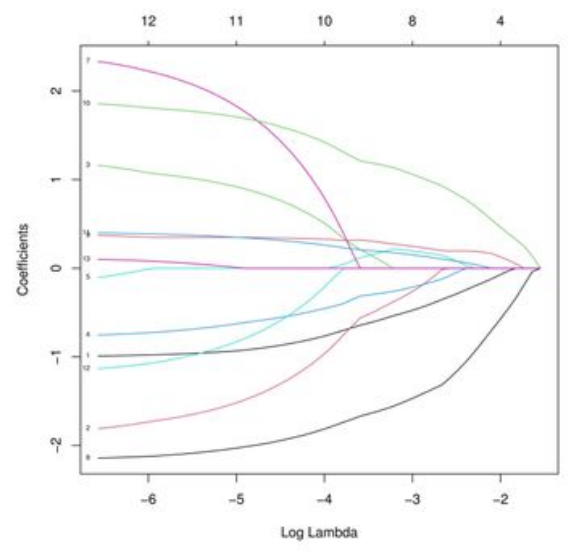

B

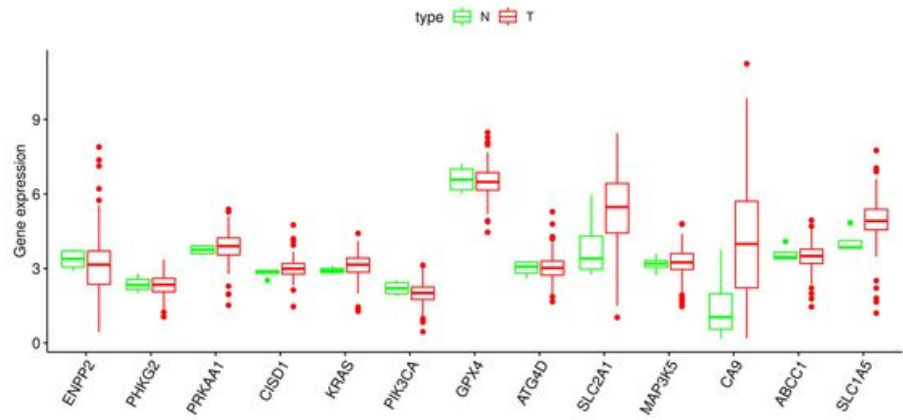

D

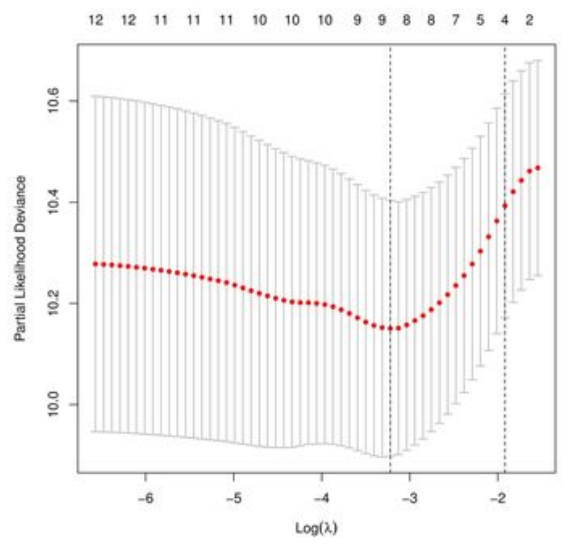

Figure 2

Screening out prognosis related FRGs for prognostic model. A Univariate Cox regression analysis to select prognosis related FRGs. B Boxplot of FRGs related to prognosis of pancreatic cancer. C The Lasso coefficient spectrum of FRGs in pancreatic cancer. D Lasso regression analysis to select the best parameters. 
A

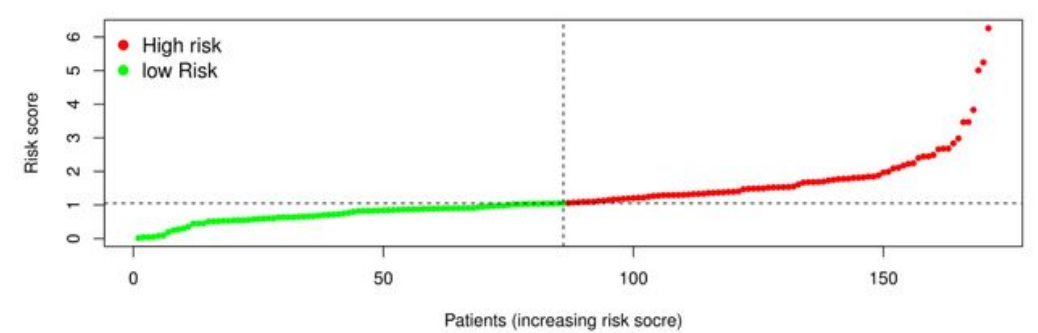

B

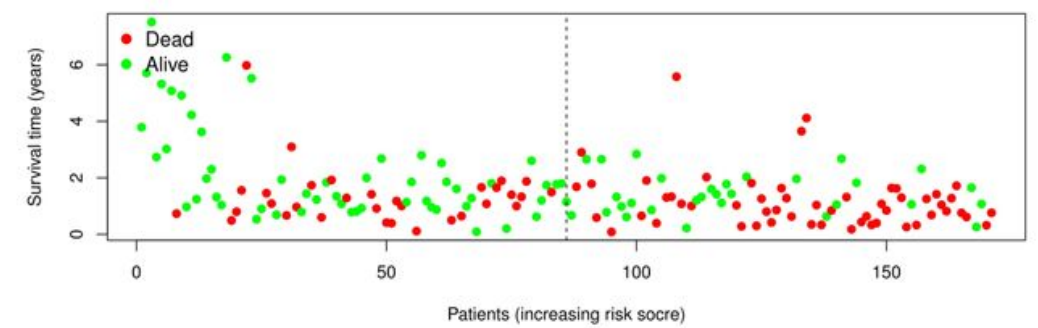

C

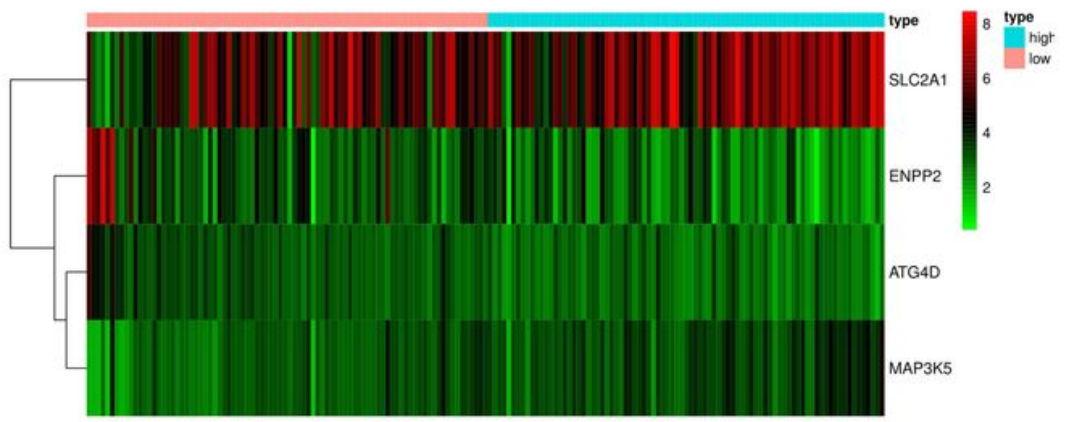

Figure 3

Characteristics of the prognostic model. A The distribution of risk scores in pancreatic cancer in TCGA. B The distribution of patient survival time and survival status. $\mathrm{C}$ Heat map of the candidate genes between the high-risk and low-risk group. 
A

$\begin{array}{lrr} & \text { pvalue } & \text { Hazard ratio } \\ \text { age } & 0.015 & 1.028(1.005-1.052) \\ \text { gender } & 0.217 & 0.762(0.495-1.173) \\ \text { grade } & 0.061 & 1.341(0.987-1.822) \\ \text { stage } & 0.214 & 1.296(0.861-1.948) \\ \mathrm{T} & 0.065 & 1.616(0.970-2.692) \\ \mathrm{N} & 0.004 & 2.233(1.292-3.857) \\ \text { riskScore } & <0.001 & 1.627(1.347-1.964)\end{array}$

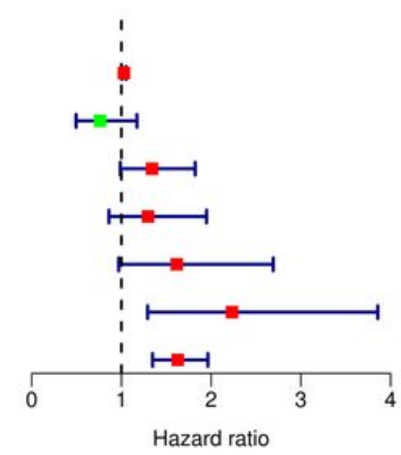

C

Risk + High risk $\mp$ Low risk

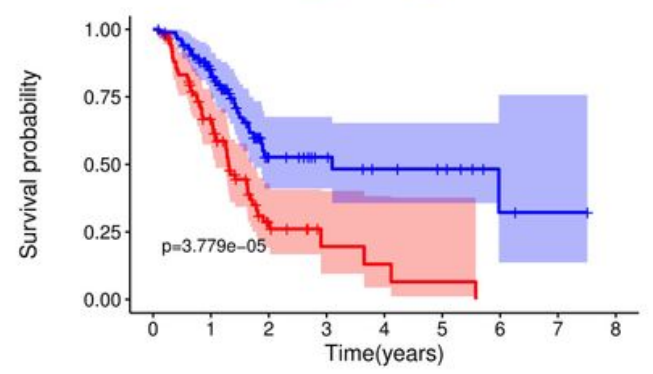

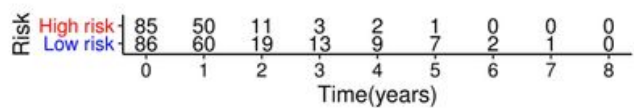

B

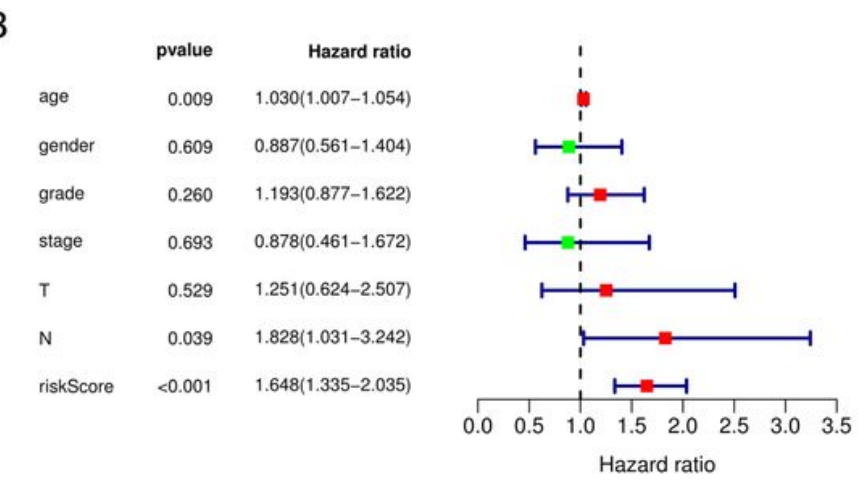

D

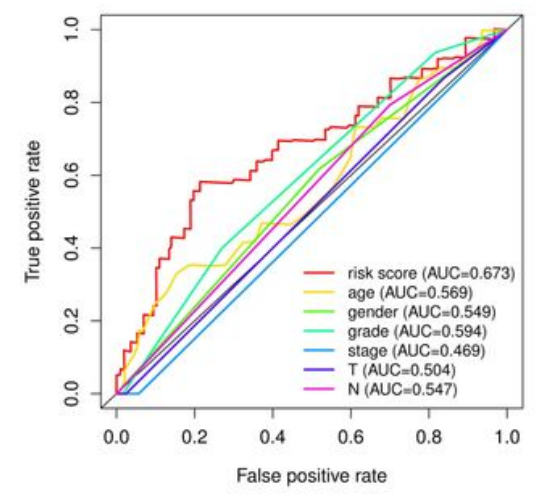

\section{Figure 4}

The prognostic model was significantly associated with survival of pancreatic cancer. A The risk score was associated with the prognosis of pancreatic cancer analyzed by univariate Cox regression analysis. $B$ The prognostic model was an independent factor of pancreatic cancer. C Kaplan-Meier curve of pancreatic cancer patients stratified by the median of risk score. The high-risk group had a poorer OS rate than did the low-risk group. D ROC analysis of the prognostic model and other clinicopathologic parameters. 
A

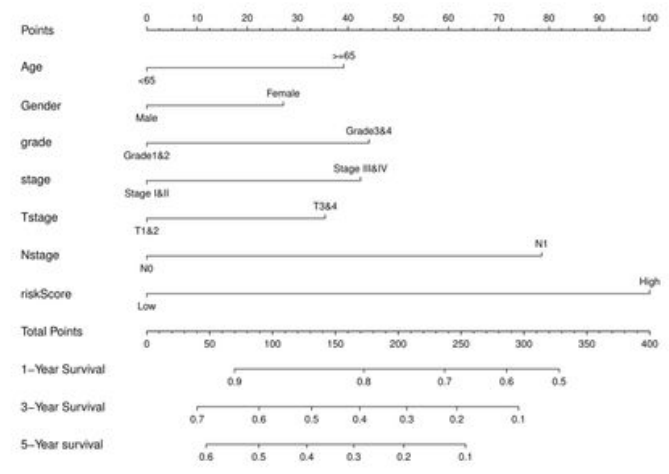

C

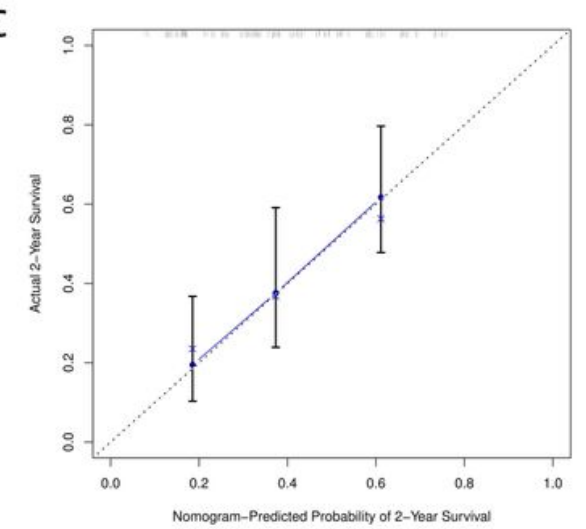

B

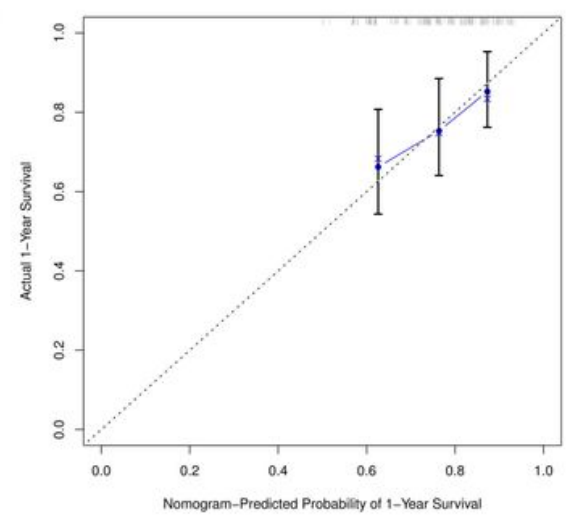

D

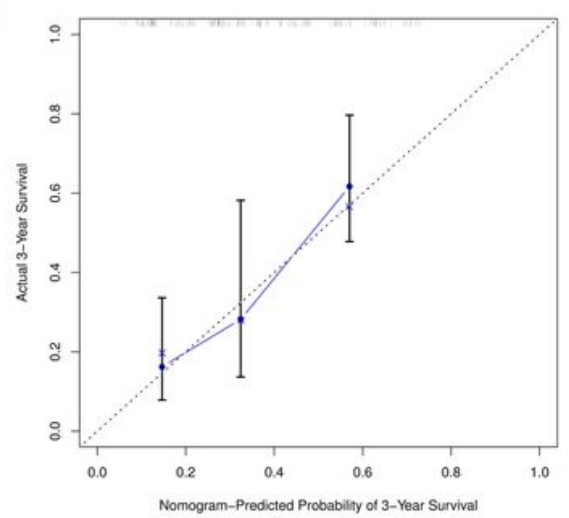

Figure 5

Construction and validation of the nomogram with the prognostic model and clinicopathological parameters. A The 1-year, 3-year and 5-year survival predicted by the nomogram based on the prognostic model. B-D Calibration plots to judge the consistence between the predicted and actual 1-year, 2-year and 3-year survival. 
A
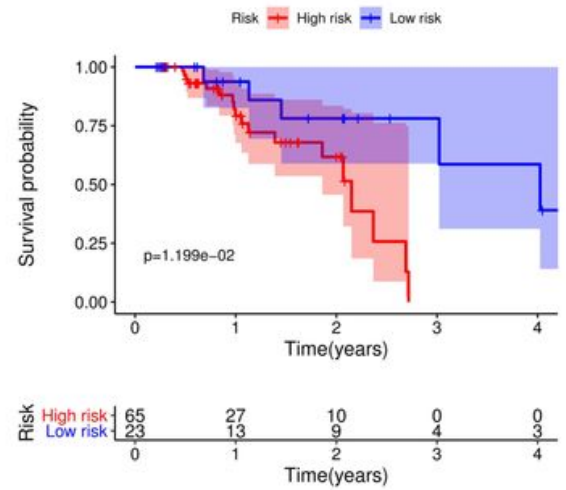

D

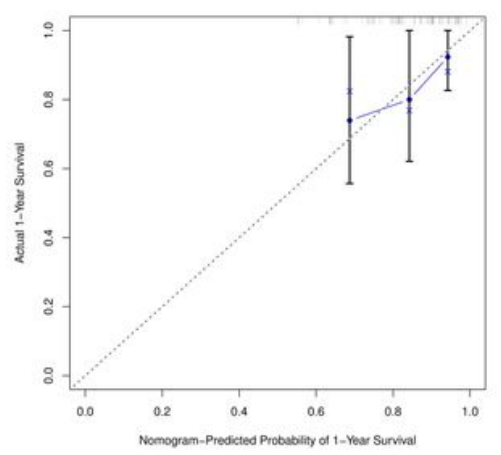

B
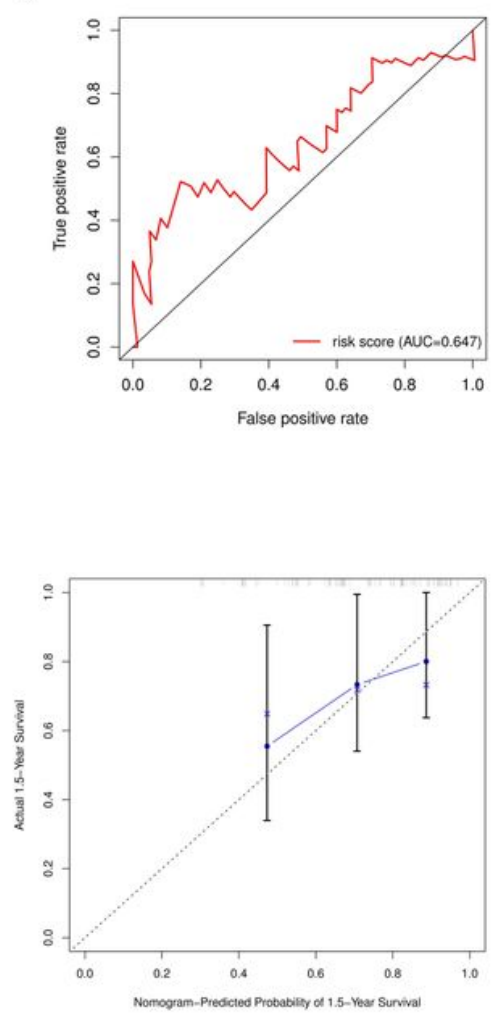

C
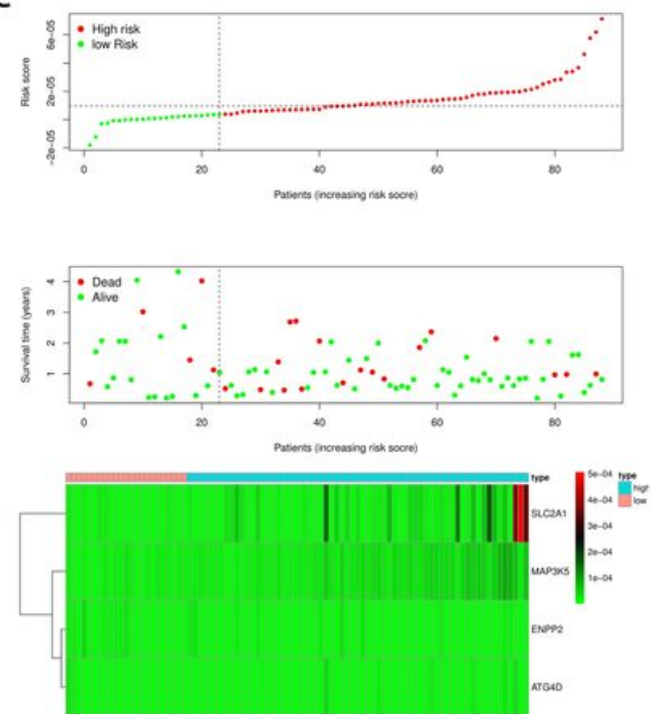

\section{Figure 6}

Experimental validation of the prognostic model. A-B Kaplan-Meier curve and ROC analysis to validate the prognostic model. C The distribution of risk score, survival time and status, and heat map between the high-risk and low-risk group. D Calibration curves to judge the consistence between the predicted and actual 1-year and 1.5-year survival. 
A

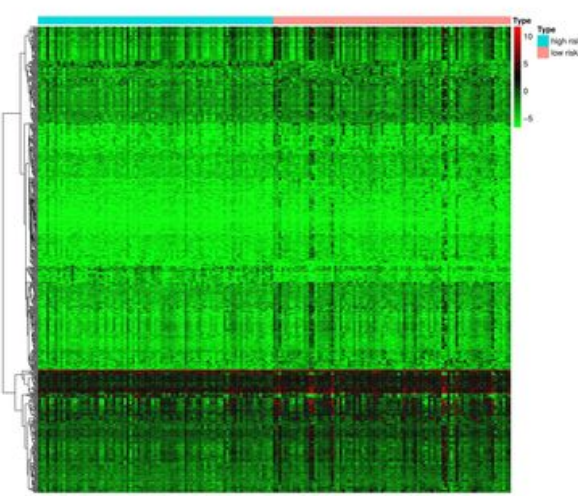

C
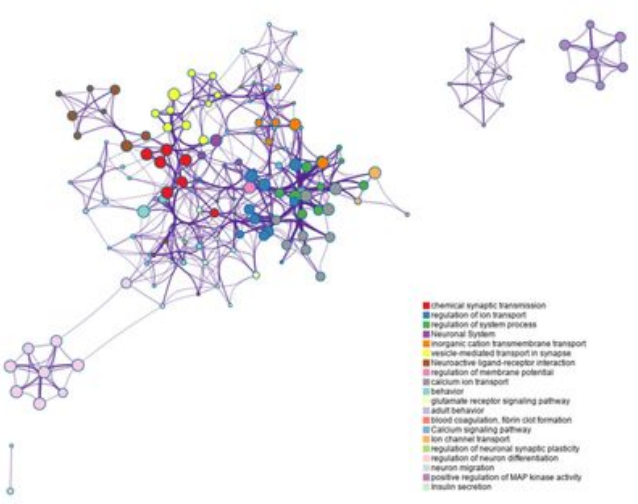

B

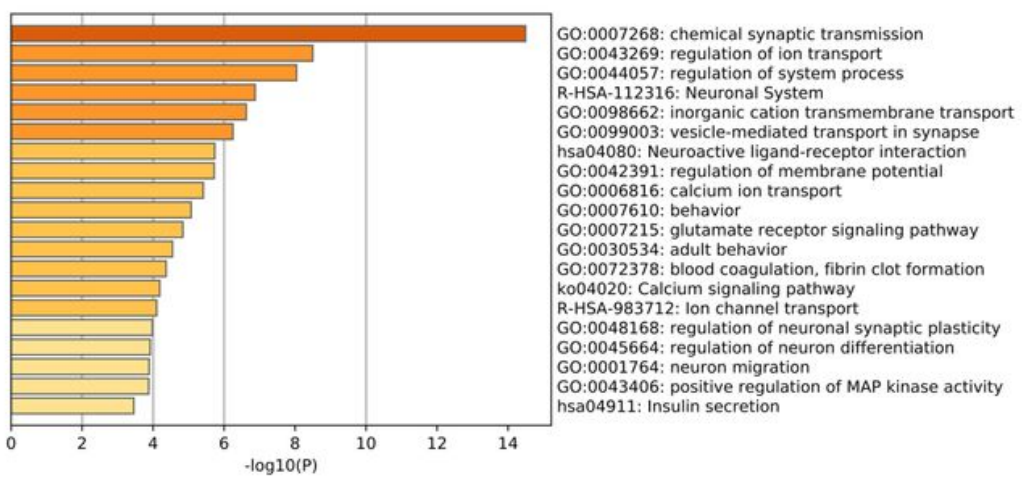

D

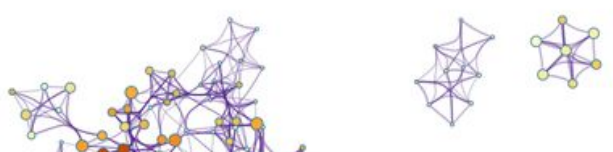

$\mathrm{E}$

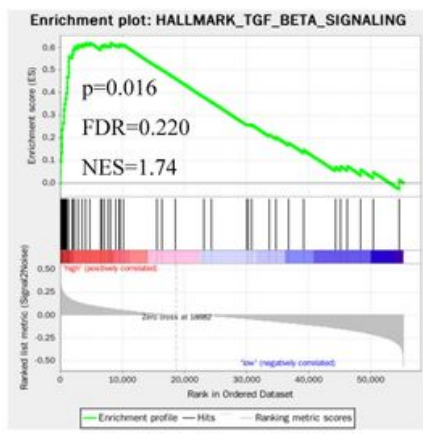

Enrichment plot: BIOCARTA_HIF_PATHWAY

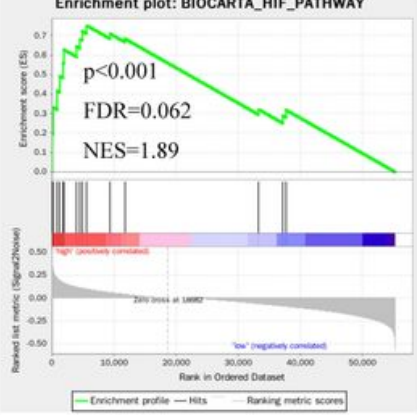

Enrichment plot: KEGG_ADHERENS_JUNCTION

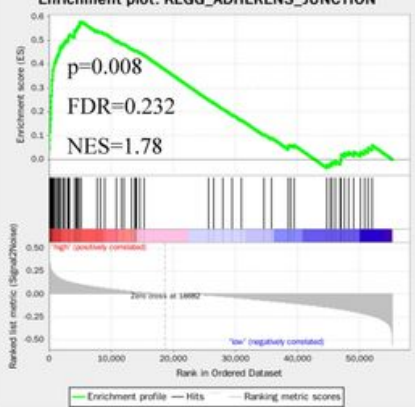

\section{Figure 7}

Enrichment analysis in the high-risk groups. A Heatmap of differentially expressed genes between highand low-risk groups. B Bar graph of the enriched terms by Metascape, colored by p-value. C Colored by cluster ID, where nodes that share the same cluster ID are typically close. D Colored by P-value, where terms containing more genes tend to have a more significant P-value. E Gene set enrichment analysis (GSEA) of the high-risk patients based on the prognostic model, including TGF $\beta$ signaling, HIF signaling pathway and adherens junction. 

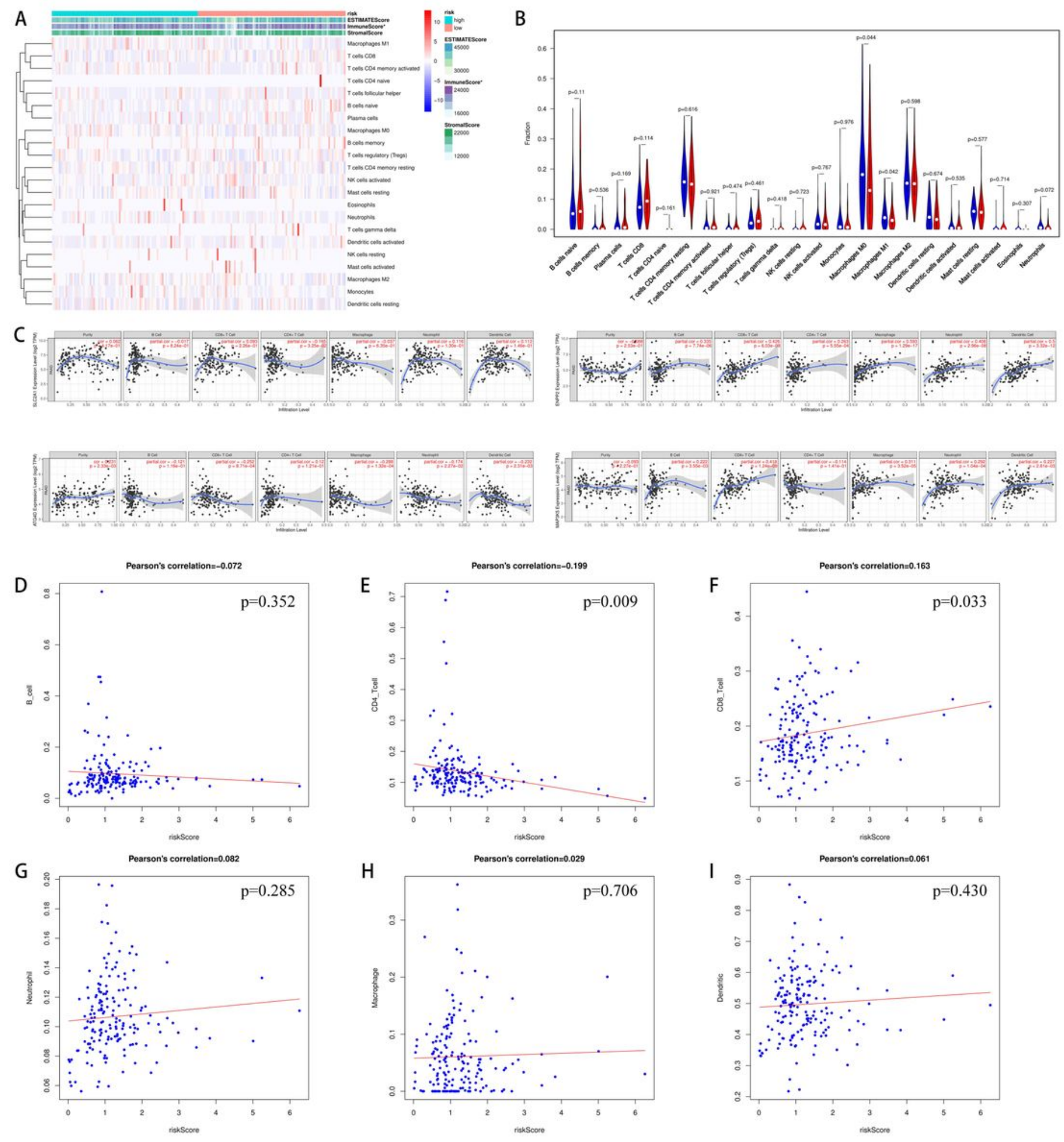

\section{Figure 8}

Risk score was associated with immune cell infiltration. A Heatmap of 22 types of immune cell infiltration level between high- and low-risk groups. B Violin plot of the difference in immune cell infiltration between high- and low-risk groups. C The four genes, including SLC2A1, ENPP2, ATG4D and MAP3K5, were associated with 6 types of immune cells by TIMER. (D-I) Relationship between risk score and B cell, CD4+T cell, CD8+T cell, neutrophil, macrophage and dendritic cell. 
A

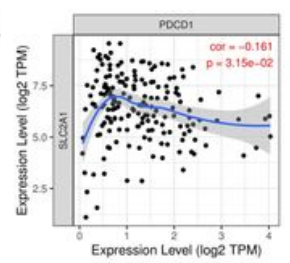

B

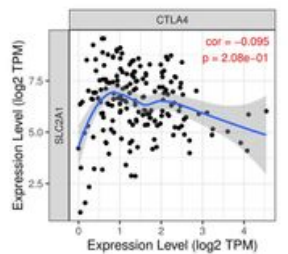

C

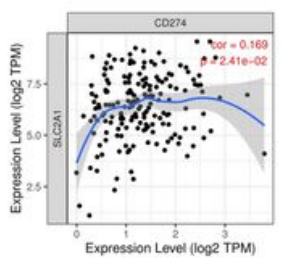

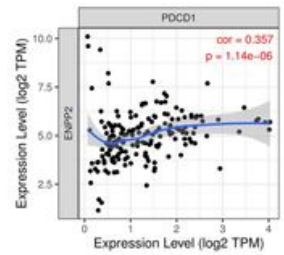
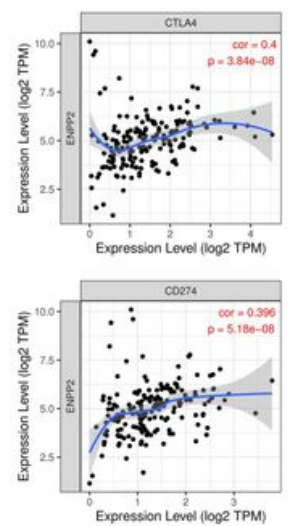
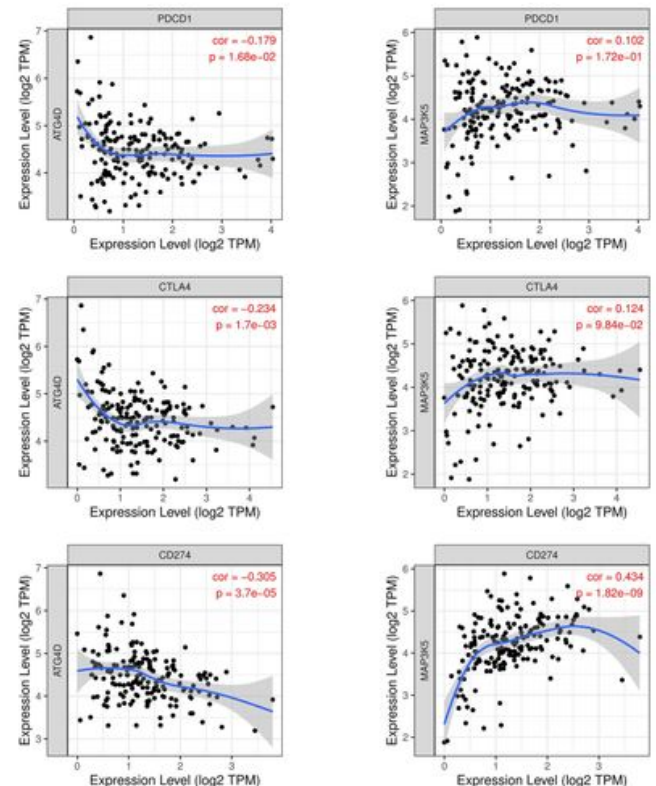
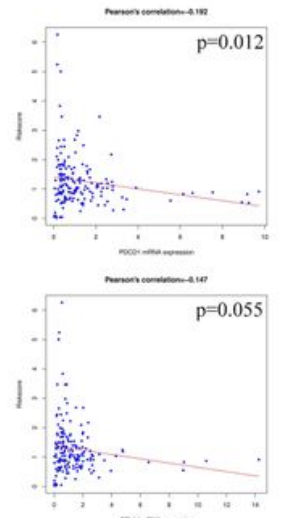

(2)

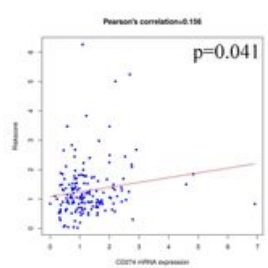

Figure 9

Risk score was associated with immune checkpoints. A PDCD1. B CTLA4. C CD274.

\section{Supplementary Files}

This is a list of supplementary files associated with this preprint. Click to download.

- supplementarymaterial.docx 